\title{
Valor Terapêutico Acrescentado
}

Carlos Alberto Fontes Ribeiro*

\section{RESUMO}

O Valor Terapêutico Acrescido (VTA) de um medicamento significa maior eficácia, segurança e/ou conveniência em relação a um comparador, que deve ser a melhor alternativa terapêutica existente. OVTA também pode significar o preenchimento de uma lacuna terapêutica, numa indicação clínica onde as terapias existentes são insuficientes, devendo nesta situação o medicamento ter apenas uma relação benefício/risco favorável. A demonstração do VTA, que é uma verdadeira inovação terapêutica que não deve ser confundida com inovação comercial ou tecnológica, deve ser feita através de metodologia robusta, obedecendo às regras da Medicina Baseada na Evidência.

Palavras-Chave: Valor Terapêutico Acrescentado; Inovação terapêutica; Medicina Baseada na Evidência.

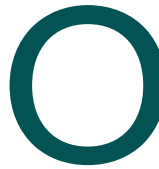
Valor Terapêutico Acrescentado ou Acrescido (VTA) resulta de avaliações comparativas entre terapêuticas com caracterização ou identificação da inovação, que podemos considerar distinta da novidade. Há preenchimento de uma lacuna ou da insuficiência terapêutica por uma nova (ou melhorada) tecnologia de saúde. OVTA pode manifestar-se por maior eficácia, maior segurança e/ou conveniência (ou aplicabilidade) do tratamento ou pela existência de terapêutica para uma entidade clínica que não a tinha (Quadro I). O custo não pode ser equacionado para o VTA, já que é calculado a partir deste.

A inovação comercial e/ou tecnológica' (Quadro II) associada a benefícios marginais não é na maioria das vezes VTA, embora possa, raramente, estar associada a vantagem terapêutica. Estas inovações que não se traduzem porVTA são quase sempre novas moléculas sem eficácia, segurança ou conveniência adicional em relação a alternativas terapêuticas já existentes.

OVTA surge, assim, com um medicamento para uma indicação clínica para a qual não existia uma opção terapêutica válida, ou existindo, a terapêutica em avaliação tem eficácia e/ou segurança superior a todas as outras alternativas.

Desde que o medicamento não seja para uma área terapêutica carenciada ou para uma indicação clínica onde exista uma lacuna terapêutica (sem terapêutica válida), e para a qual demonstra eficácia, a avaliação da eficácia acrescida tem de ser feita relativamente a um

*Professor de Farmacologia da Faculdade de Medicina da Universidade de Coimbra; Farmacologista Clínico.
QUADRO I. Conceito de Valor Terapêutico Acrescentado (VTA)

- Deve ser traduzido pela maior:

Eficácia

Segurança

e/ou Conveniência

- Tratamento de uma indicação clínica para a qual não existia uma opção terapêutica válida

comparador activo (na lacuna terapêutica a comparação é feita com o não tratamento). Nestas circunstâncias há geralmente um problema que é a inexistência de ensaios clínicos com uma comparação directa entre o medicamento em avaliação e o comparador que tem demonstrado a melhor relação benefício / risco. Quase todos os ensaios clínicos são feitos versus placebo ou então é escolhido um medicamento que não é o mais apropriado. Van Luijn et al. ${ }^{2}$ referem que entre $1999 \mathrm{e}$ 2005 apenas $48 \%$ dos medicamentos aprovados na União Europeia tinham sido estudados comparativamente a outros medicamentos. Destes, apenas cerca de 1/3 dos ensaios clínicos haviam sido publicados. Este panorama dos ensaios clínicos das fases II e III não se altera com os estudos clínicos da fase IV, ainda menos vezes feitos. Os estudos de efectividade são escassos e a maioria dos estudos pós-AIM dizem respeito ao estudo da segurança.

Deste modo, a comparação tem de ser indirecta [por exemplo, entre os medicamentos A e B pelo ganho terapêutico ou absoluto de $\mathrm{A}$ (eficácia de $\mathrm{A}$ menos a efi- 


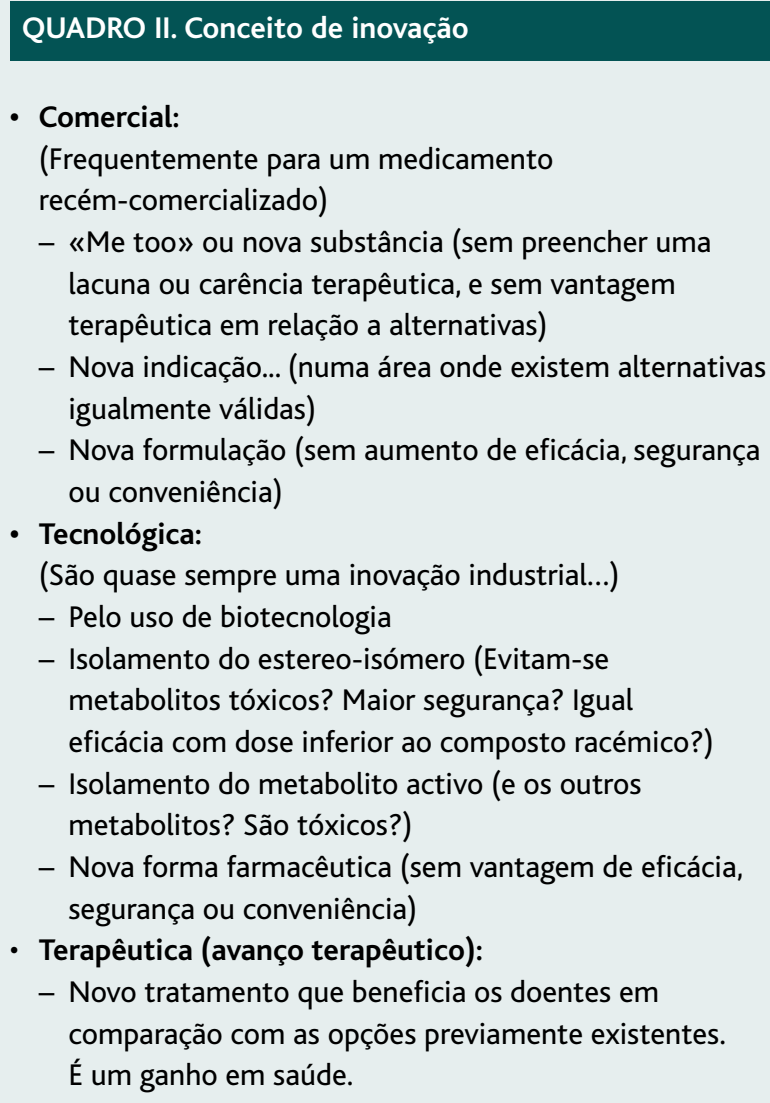

cácia do placebo) comparado com o do fármaco B (eficácia de B menos a eficácia do placebo)] e, geralmente, as populações e as circunstâncias dos diferentes ensaios clínicos não são idênticas. Outras vezes, também existe o problema de o medicamento comparador ser antigo e não ter o suporte de ensaios clínicos robustos. Na minha opinião deverá ser usado o medicamento com o uso clínico melhor estabelecido, embora tal fuja às regras da Medicina Baseada na Evidência (porém, esta regra do uso bem estabelecido é aceite para a AIM). Por outro lado, muitas vezes o horizonte temporal dos medicamentos em comparação não é similar ou nem sequer o mais apropriado - quando se faz a avaliação doVTA de um medicamento não existem ainda estudos de efectividade, excepto em raras situações (ao contrário do que muitas vezes se pensa e diz, um ensaio de grandes dimensões ou vários ensaios clínicos não comprovam a efectividade!).
QUADRO III. Valor terapêutico acrescentado pela maior segurança

- Exposição populacional ao medicamento suficiente? DDDs?

- Exposição temporal? Quantos meses ou anos?

- Tipo de população em que foi utilizado o medicamento?

- Raças? Grupos de risco? Interacções farmacológicas e outras?

- Sistemas de notificação de reacções adversas?

- Por exemplo, houve indicação para se notificarem apenas as reacções adversas graves e não esperadas? A não notificação no nosso país significa que o medicamento é mais seguro?

- Estão implementados sistemas de qualidade do sistema e dos métodos usados na Farmacovigilância? Suporte económico para a implementação da Farmacovigilância?

- Informação e formação em Farmacovigilância?

DDD: Dose Diária Definida

Quanto à segurança (Quadro III), um medicamento só poderá ser superior a outro (quando as diferenças não são muito acentuadas) quando houver exposição suficiente (número adequado de indivíduos, que pode ser medido através das DDD (doses diárias definidas, que são as doses diárias médias para a indicação clínica major consumidas do medicamento em avaliação) e tempo suficiente de exposição (um ensaio de 12 semanas com um antihipertensor é suficiente? Seis meses para um medicamento para tratar a osteoporose ou a demência de Alzheimer é suficiente?).

Quanto à conveniência - talvez o factor mais vezes invocado como valor acrescido -, tem de haver demonstração sólida de superioridade (com o medicamento em avaliação e não com a extrapolação de dados de outros medicamentos). Embora uma nova forma farmacêutica tenha maior rapidez de acção e menor latência, para o efeito é necessário evidenciar que tal é clinicamente relevante e significativo [por exemplo, um comprimido orodispersível é vantajoso para a maioria dos doentes? É mais rápido que um comprimido deglutido na via oral? (os triptanos orodispersíveis não o são!) Ou é uma forma de «fugir» ao genérico sem qualquerVTA? A própria forma orodispersível não pode 
desencadear náuseas e/ou vómitos?]. A comodidade não deve prejudicar a eficácia (por exemplo, os sistemas transdérmicos em relação a formas orais têm a mesma eficácia em qualquer circunstância? E com a transpiração? A diminuição do número de tomas diárias aumenta a adesão? Foi demonstrado para o medicamento em questão (ao diminuir o numero de tomas diárias pode-se ter aumentado a dose e haver uma reacção adversa de pico de dose, por exemplo)? Uma toma oral mensal é superior a uma toma semanal? Foi demonstrado? Qual é a importância de se esquecer ou de tomar uma dose dupla na toma mensal? Não haverá sub ou sobredosagem com a confusão na toma? As formas de libertação controlada podem permitir concentrações sanguíneas com menor flutuação, mais estáveis, mas o efeito do fármaco pode depender do Cmax (ou concentração máxima atingida ou pico de concentração) ou estas formas podem ser prejudicadas pelo trânsito intestinal acelerado ou pode haver demora na obtenção da Css (concentração de «steady-state» ou de patamar). Ou seja, é necessário demonstrar a vantagem ou o eventual VTA!

Tendo em atenção estes conceitos torna-se claro que a inovação comercial raramente significa inovação terapêutica (Quadro II), que é um ganho em Saúde. Um medicamento recém-comercializado é muitas vezes um «me-too», ou uma nova substância que não vem preencher nenhuma lacuna terapêutica ou que não tem maior eficácia ou melhor segurança do que as alternativas terapêuticas existentes para a indicação clínica (incluindo as novas indicações clínicas para o medicamento em avaliação), ou uma nova formulação sem aumento de eficácia, segurança ou conveniência.

Há uma grande confusão entre Autorização de Introdução no Mercado (AIM) e VTA. Para ter AIM o medicamento apenas tem de demonstrar uma razão benefício / risco aceitável. Não tem de demonstrarVTA! Habitualmente este é avaliado em sede de comparticipação ou de avaliação de medicamentos para uso exclusivo hospitalar. Existem muitos medicamentos comercializados que não apresentam VTA em relação a outras alternativas terapêuticas!

Outro tipo de inovação é a tecnológica ou industrial (Quadro II), raramente constituindo-se como verdadeiro avanço terapêutico, e que pode ser feita através de: - Uso de biotecnologia (traz medicamentos inovado- res terapêuticos?);

- Obtenção de estereoisómeros activos sem significado clínico (evitam-se metabolitos tóxicos? Há maior segurança? Não há conversão de um estereoisómero noutro estereoisómero com aumento da forma racémica? (por exemplo, o esomeprazole não se converte no omeprazole?);

- Isolamento de metabolitos activos (o fármaco original tinha uma relação benefício/risco problemática? (Por exemplo, a bromexina foi ultrapassada pelo ambroxol, seu metabolito activo? Havia um problema de eficácia e segurança com a bromexina?);

- Desenvolvimento de novas formas farmacêuticas sem vantagem na eficácia, segurança ou conveniência.

O termo «inovação incremental» resulta em parte do conceito associado ao incremento na análise de custo-efectividade, calculado de ensaios clínicos. Pode ser um conceito enganador e pode (quase sempre) não significar VTA.

Existem, porém, numerosos medicamentos que são inovações tecnológicas e comerciais e que também representam inovações terapêuticas. A este propósito é necessário dizer que um novo mecanismo de acção pode não constituir um avanço terapêutico, desde que as consequências clínicas do uso do novo medicamento sejam as mesmas das alternativas existentes, o que acontece com muita frequência.

Finalmente, uma chamada de atenção para os ensaios clínicos que para avaliar o VTA devem ser robustos (para o novo medicamento) (Quadro IV) ou então o medicamento deve ter a prova do tempo ou estudos de efectividade (para os comparadores, desde que não tenha os referidos ensaios clínicos).

Assim, os ensaios clínicos, aleatorizados e controlados (RCTs), devem ter uma amostra suficiente, calculada [tendo em conta os falsos positivos e negativos, a eficácia esperada e a taxa de dropouts (ou taxa de desistências ou taxa de erosão)], devem ter objectivos clinicamente relevantes, finais (ou substitutivos em determinadas condições), devem ter um desenho apropriado (para uma associação fixa o ensaio tem de ter um desenho factorial), duração suficiente (quando eticamente não o pode ser, deve ter extensões, de modo a considerar a história natural da doença), colheita fidedigna de dados (obrigatoriamente em boas práticas clí- 


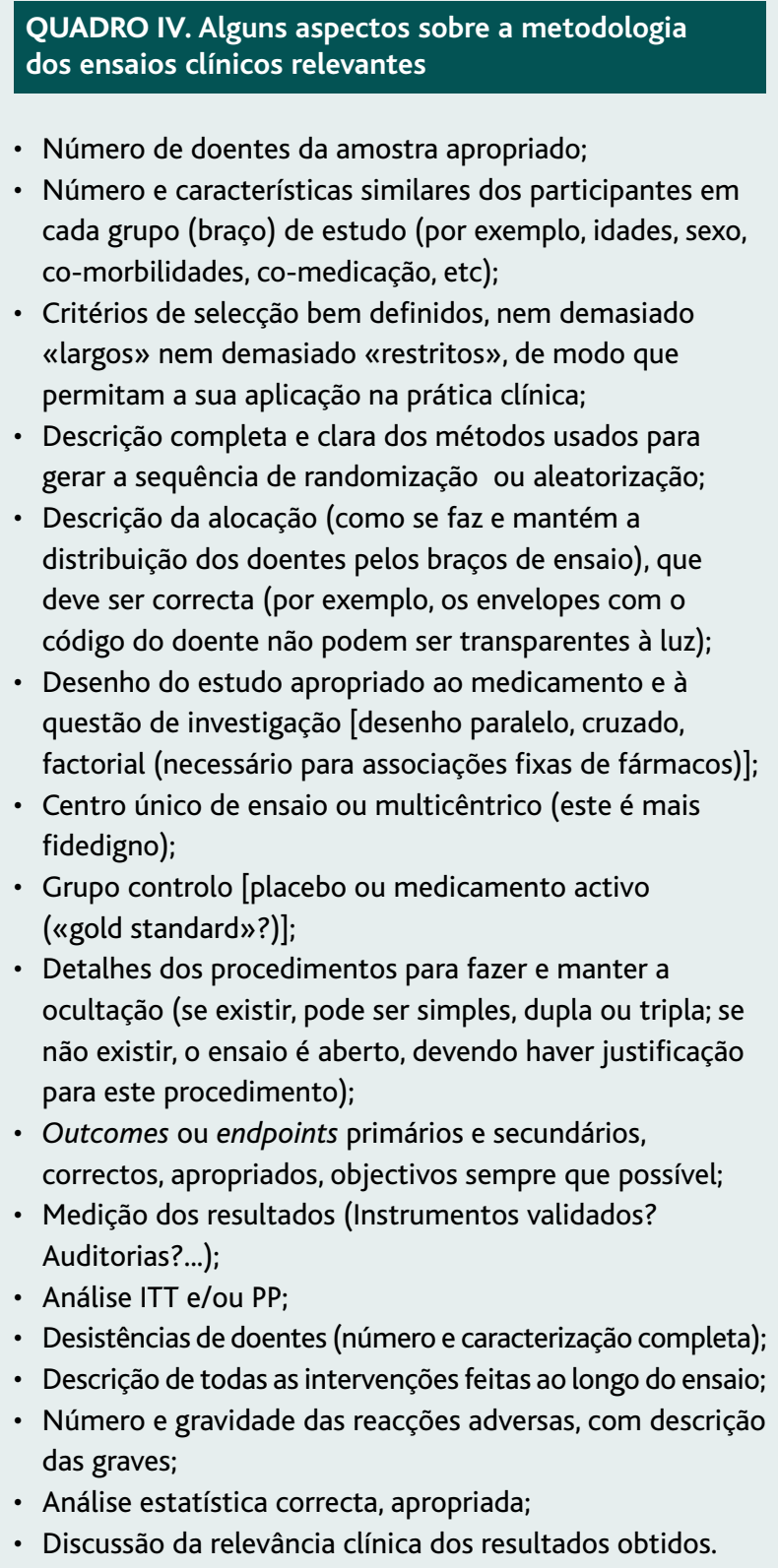

- Número de doentes da amostra apropriado;

- Número e características similares dos participantes em cada grupo (braço) de estudo (por exemplo, idades, sexo, co-morbilidades, co-medicação, etc);

- Critérios de selecção bem definidos, nem demasiado «largos» nem demasiado «restritos», de modo que permitam a sua aplicação na prática clínica;

- Descrição completa e clara dos métodos usados para gerar a sequência de randomização ou aleatorização;

- Descrição da alocação (como se faz e mantém a distribuição dos doentes pelos braços de ensaio), que deve ser correcta (por exemplo, os envelopes com o código do doente não podem ser transparentes à luz);

- Desenho do estudo apropriado ao medicamento e à questão de investigação [desenho paralelo, cruzado, factorial (necessário para associações fixas de fármacos)];

- Centro único de ensaio ou multicêntrico (este é mais fidedigno);

- Grupo controlo [placebo ou medicamento activo («gold standard»?)];

- Detalhes dos procedimentos para fazer e manter a ocultação (se existir, pode ser simples, dupla ou tripla; se não existir, o ensaio é aberto, devendo haver justificação para este procedimento);

- Outcomes ou endpoints primários e secundários, correctos, apropriados, objectivos sempre que possível;

- Medição dos resultados (Instrumentos validados? Auditorias?...);

- Análise ITT e/ou PP;

- Desistências de doentes (número e caracterização completa);

- Descrição de todas as intervenções feitas ao longo do ensaio;

- Número e gravidade das reacções adversas, com descrição das graves;

- Análise estatística correcta, apropriada;

- Discussão da relevância clínica dos resultados obtidos.

Análise ITT: análise em intenção de tratar, ou de todos os casos que iniciaram o tratamento, independentemente de o continuarem. Análise PP: análise per protocol, ou de apenas os casos que concluíram o ensaio ou tratamento.

nicas e em boas práticas de laboratório, quando estas últimas se justifiquem), análise correcta dos dados [em PP («per protocol» ou análise final dos doentes que chegaram ao fim do ensaio) e ITT (em intenção de tratar ou análise de todos os doentes que iniciaram a terapêutica)]; o ensaio deve ter ainda validade interna e exter- na (os critérios de inclusão e exclusão não podem tornar muito diferentes os doentes do ensaio em relação aos doentes da prática clínica). Os resultados devem ser clinicamente relevantes - cuidado com a cultura do «p»! A determinação da eficácia e segurança deve ter em conta o conhecimento sobre o fármaco (por exemplo, se os canabinóides têm efeitos analgésicos e antieméticos é racional que o ensaio clínico de um antagonista dos canabinóides (como o antagonista dos receptores $\mathrm{CB} 1$, o rimonabant) pesquise o eventual aumento de dor, e de estados dolorosos, e de náuseas e vómitos).

Os marcadores usados para medir a expressão da doença ou do sinal/sintoma são frequentemente controversos. O ideal seria o uso de marcadores/endpoints finais da doença (por exemplo, complicações micro e macrovasculares da diabetes mellitus do tipo 2) mas como tal só seria possível ao fim de muitos anos de tratamento, o que habitualmente se usa são marcadores/endpoints substitutivos ou «surrogados» (por exemplo, glicemias ou, melhor, HbAlC glicada na diabetes mellitus do tipo 2). Todavia, tem de haver uma forte correlação entre o marcador substitutivo utilizado e o marcador final da doença [por exemplo, a HbA1C glicada persistentemente elevada correlaciona-se com as complicações micro e macrovasculares; porém, já é discutível e controverso se a densidade mineral óssea (DMO) - marcador substitutivo - se correlaciona com as fracturas vertebrais e extravertebrais (marcador final) na osteoporose]. O marcador substitutivo deve ter as qualidades expressas no Quadro V e existem marcadores dos tipos descritos no Quadro VI.

Em Farmacologia, uma das mais importantes características de um fármaco é a dose-dependência. Na avaliação do VTA esta qualidade deve ser tida em conta, porque pode avaliar de modo negativo doses elevadas de um medicamento - duplicando a dose duplicou-se

QUADRO V. Características de um marcador substitutivo (ou «surrogado»).

Fiável, reprodutível, facilmente mensurável, com efeito dose-resposta nítido, preditivo em relação à doença (relação bem estudada com a doença), plausível biologicamente, sensível, específico, bem caracterizado (com valores de referência bem determinados), sujeito a controlo de qualidade 


\section{QUADRO VI. Tipos de marcadores de doença}

- Bioquímicos

- Fisiopatológicos

- Anátomo-patológicos

- Clínicos

- Primários ou secundários?

- Marcador primário

- Marcador substitutivo («surrogate endpoint»)

- Horizonte temporal? (eficácia vs efectividade)

- Eficácia mantida? Consistente?

- Medição? Valor diferencial aceitável?

Clinicamente relevante?

- Se a avaliação for através de escalas, estas estão validadas para a população a estudar? Medem o que se pretende medir? Sensibilidade e especificidade?

Reprodutibilidade?..

o efeito? Ou há a possibilidade de mais reacções adversas sem haver proporcionalidade para a eficácia? Como é a dose-dependência para as estatinas ou para os ARAs II? É evidente?

No Quadro VII estão indicados alguns dos marcadores usados para medir o efeito ou eficácia de um medicamento.

Em conclusão, o Valor Terapêutico Acrescentado ou Acrescido traduz-se pelo preenchimento de uma lacuna ou carência terapêutica ou por uma maior eficácia/efectividade, segurança e/ou conveniência. É um ganho em Saúde que devemos privilegiar e aplicar, tanto a nível regulamentar como na prática clínica!

\section{REFERÊNCIAS BIBLIOGRÁFICAS}

1. Pouvourville G. Risk-sharing agreements for innovative drugs: a new solution to old problems? Eur J Health Econ 2006 Sep; 7 (3): 155-7.

2. Van Luijn JC, Gribnau FW, Leufkens HG. Availability of comparative trials for the assessment of new medicines in the European Union at the moment of market authorization. Br J Clin Pharmacol 2007 Feb; 63 (2): 159-62.

\section{LEITURAS ACONSELHADAS}

National Institute for Clinical Excellence. Guide to the Technology Appraisal Process; April 2004. Disponível em: http://www.nice.org.uk [acedido em 22/12/2008].
QUADRO VII. Alguns marcadores possíveis de eficácia em ensaios clínicos aleatorizados e controlados

- Tempo (dias, meses), \% de melhoria de uma determinada variável

- Intervalo de recorrência de sintomas após paragem do tratamento (retorno a $75 \%$ ou $80 \%$ da linha de base, etc)

- Grau de recorrência dos sintomas após cessação do tratamento

- Intervalo de tempo, sob tratamento activo, até um novo episódio da doença aparecer

- Duração da melhoria clínica sob tratamento de manutenção

- Magnitude do efeito terapêutico em intervalos de tempo determinados após início do tratamento (por ex., 7 dias, 30 dias, 180 dias)

- Parâmetros de eficácia calculados com base em marcadores substitutivos (fracção de ejecção, pressão ventricular no fim da diástole, etc)

- Parâmetros subjectivos de melhoria do doente

- Parâmetros relacionados com a qualidade de vida

- Melhoria ou desaparição de factores de risco de doença

- Mortalidade geral e específica de doença

- Morbilidade (reacções adversas, demora hospitalar média, etc)

- Impressão clínica global do estado de saúde do doente (pelo médico ou pelo doente)

- Intervalo de tempo entre episódios de doença (convulsões, por exemplo)

- Taxa de dropouts do estudo

- Necessidade de terapêutica concomitante

- Prevenção da deterioração física provocada pela doença

Jadad A. Randomised controlled trials. London: BMJ Books; 1998.

Lesko SM, Mitchell AA. The use of randomized controlled trials for pharmacoepidemiologic studies. In: Stom BL, Kimmel SE, editors. Textbook of Pharmacoepidemiology. West Sussex: John Wiley \& Sons; 2006. p. 311-20.

Mayer D. Essential Evidence-Based Medicine. Cambridge: Cambridge University Press; 2004.

Prescrire International. European Medicines Policy, June 2002. Disponível em: http://www.prescrire.org [acedido em 22/12/208].

- Strom, B. The use of pharmacoepidemiology to study beneficial drug effects. In: Stom BL, Kimmel SE, editors. Textbook of Pharmacoepidemiology. West Sussex: John Wiley \& Sons; 2006. p. 321-31.

\section{ENDEREÇO PARA CORRESPONDÊNCIA}

Carlos Alberto Fontes Ribeiro

E-mail: fontes.ribeiro@gmail.com 


\section{ABSTRACT}

The Added Therapeutic Value (ATV) means a better efficacy, safety and / or convenience of a drug in comparison with the best therapeutic alternative till now. ATV can also signify the drug effect with an acceptable benefit / risk ratio to a therapeutic necessity in a clinical indication without drugs. The demonstration of the ATV, which is a real therapeutic innovation and not only a commercial or technological innovation, must be performed through the Evidence-Based Medicine methodologies.

Keywords: Added Therapeutic Value; Therapeutic Innovation; Evidence-Based Medicine. 